\title{
Efficient Kernel Density Estimation Using the Fast Gauss Transform with Applications to Segmentation and Tracking
}

\author{
Ahmed Elgammal, Ramani Duraiswami, Larry S. Davis \\ Computer Vision Laboratory \\ The University of Maryland \\ College Park, MD 20742, USA
}

\begin{abstract}
The study of many vision problems is reduced to the estimation of a probability density function from observations. Kernel density estimation techniques are quite general and powerful methods for this problem, but have a significant disadvantage in that they are computationally intensive. In this paper we explore the use of kernel density estimation with the fast gauss transform (FGT) for problems in vision. The FGT allows the summation of a mixture of $M$ Gaussians at $N$ evaluation points in $O(M+N)$ time as opposed to $O(M N)$ time for a naive evaluation, and can be used to considerably speed up kernel density estimation. We present applications of the technique to problems from image segmentation and tracking, and show that the algorithm allows application of advanced statistical techniques to solve practical vision problems in real time with today's computers.
\end{abstract}

\section{Introduction}

Many problems in computer vision can be posed as obtaining the probability density function describing an observed random quantity. In general the forms of the underlying density functions are not known. While classical parametric densities are mostly unimodal, practical computer vision problems involve multimodal densities. Further, high-dimensional densities can not often be simply represented as the product of one-dimensional density functions. While mixture methods partially alleviate this problem, they require knowledge about the problem to choose the number of mixture functions, and their individual parameters.

An attractive feature of nonparametric procedures is that they can be used with arbitrary distributions and without the assumption that the forms of the 
underlying densities are known. However, most nonparametric methods require that all of the samples be stored or that the designer have extensive knowledge of the problem. Since a large number of samples is needed to obtain good estimates, the memory requirements can be severe [1].

A particular nonparametric technique that estimates the underlying density and is quite general is the kernel density estimation technique. In this technique the underlying probability density function is estimated as

$$
\hat{f}(x)=\sum_{i} \alpha_{i} K\left(x-x_{i}\right)
$$

where $K$ is a "kernel function" (typically a Gaussian) centered at the data points, $x_{i}, i=1 . . n$ and $\alpha_{i}$ are weighting coefficients (typically uniform weights are used, i.e., $\left.\alpha_{i}=1 / n\right)$. Note that choosing the Gaussian as a kernel function is different from fitting the distribution to a Gaussian model. Here, the Gaussian is only used as a function that is weighted around the data points. The use of such an approach requires a way to efficiently evaluate the estimate $\hat{f}\left(x_{j}\right)$ at any new point $x_{j}$. A good discussion of kernel estimation techniques can be found in [2].

In general, given $N$ original data samples and $M$ points at which the density must be evaluated, the complexity is $O(N M)$ evaluations of the kernel function, multiplications and additions. For many applications in computer vision, where both real-time operation and generality of the classifier are desired, this complexity can be a significant barrier to the use of these density estimation techniques. In this paper we discuss the application of an algorithm, the Fast Gauss Transform (FGT) [3, 4], that improves the complexity of this evaluation to $O(N+M)$ operations, to computer vision problems.

The FGT is one of a class of very interesting and important new families of fast evaluation algorithms that have been developed over the past dozen years, and are revolutionizing numerical analysis. These algorithms use the fact that all computations are required only to a certain accuracy (which can be arbitrary) to speed up calculations. These algorithms enable rapid calculation of approximations of arbitrary accuracy to matrix-vector products of the form Ad where $a_{i j}=\phi\left(\left|x_{i}-x_{j}\right|\right)$ and $\phi$ is a particular special function. These sums first arose in applications that involve potential fields where the functions $\phi$ were spherical harmonics, and go by the collective name "fast multipole" methods [3]. The basic idea is to cluster the sources and target points using appropriate data structures, and to replace the sums with smaller summations that are equivalent to a given level of precision. Interesting applications of these algorithms include fast versions of Fourier transforms that do not require evenly spaced data points [5]. An algorithm for evaluating Gaussian sums using this technique was developed by Greengard and Strain [4]. We will be concerned with this algorithm and its extensions in this paper. Related work also includes the work by Lambert et al [6] for efficient on-line computation of kernel density estimation.

Section 2 introduces some details of the Fast Gauss Transform, and our improvements to it. We also present practical demonstrations of the improvement 
in complexity. In Section 3 we introduce using kernel density estimation to model the color of homogenous regions and use this approach to segment foreground region corresponding to people into major body parts. In Section 4 we show how the fast Gauss algorithm can be used to efficiently compute estimates for density gradient and use this for tracking people. The tracking algorithm is robust to partial occlusion and rotation in depth and can be used with stationary or moving cameras. Appropriate reviews of the relevant related work are provided in these self-contained sections.

\section{Fast Gauss Transform}

The FGT was introduced by Greengard and Strain $[3,4]$ for the rapid evaluation of sums of the form

$$
S\left(t_{i}\right)=\sum_{j=1}^{N} f_{j} \exp \left(-\frac{\left(s_{j}-t_{i}\right)^{2}}{\sigma^{2}}\right), \quad i=1, \ldots, M .
$$

Here $s_{j}$ and $t_{i}$ are respectively the $d$-dimensional "source" and "target" coordinates, while $\sigma$ is a scalar and $f_{j}$ are source strengths. They showed that using their fast algorithm this sum could be computed in $O(N+M)$ operations. They also showed results from 1-D and 2-D tests of the algorithm. It was extended by Strain [7] to sums where $\sigma$ in equation 2 varied with the position of the target or the source, i.e., for the case where $\sigma$ depends on the source location the sum is

$$
S\left(t_{i}\right)=\sum_{j=1}^{N} f_{j} \exp \left(-\frac{\left(s_{j}-t_{i}\right)^{2}}{\sigma_{j}^{2}}\right), \quad i=1, \ldots, M .
$$

A puzzling aspect of the FGT is that even though the algorithm was published fourteen years ago, and Gaussian mixture sums arise often in statistics (as already noted by Greengard and Strain), the algorithm has not been used much in applications. Subsequent publications on the FGT have only begun to appear again over the last two years.

An important reason for the lack of use of the algorithm is probably the fact that it is inconvenient to do so. First, it is not immediately clear what the cross-over point is when the algorithm begins to manifest its superior asymptotic complexity and offsets the pre-processing overhead. While the nominal complexity of the algorithm is $O(M+N)$, the constant multiplying it is $O\left(p^{d}\right)$, where $p$ is the number of retained terms in a polynomial approximation (described below). This makes it unclear if the algorithm is useful for higher dimension applications seen in statistical pattern recognition. The fact that there is no readily available implementation to test these issues acts as a further barrier to its wide adoption.

Another reason for the delay in applying the FGT to problems in computational statistics might be that the algorithm, as originally presented, are not directly applicable to general mixture of gaussian sums that statisticians are 
used to thinking about. General $d$-dimensional mixture of gaussian sums have the form

$$
S\left(t_{i}\right)=\sum_{j=1}^{N} f_{j} \exp \left(-\left(t_{i}-s_{j}\right)^{\prime} V^{-1}\left(t_{i}-s_{j}\right)\right)
$$

with $V^{-1}$ is a symmetric positive definite covariance matrix, which is much more complex than 2. Even in the case of kernel density estimation with Gaussians, the form of the Gaussian sum encountered is

$$
S\left(t_{i}\right)=\sum_{j=1}^{N} f_{j} e^{-\sum_{k=1}^{d}\left(\frac{\left(t_{i}-s_{j}\right)_{k}}{\sigma_{k}}\right)^{2}}=\sum_{j=1}^{N} f_{j} e^{-\left[\left(\frac{\left(t_{i}-s_{j}\right)_{1}}{\sigma_{1}}\right)^{2}+\ldots+\left(\frac{\left(t_{i}-s_{j}\right)_{d}}{\sigma_{d}}\right)^{2}\right]}
$$

where the subscript $k$ indicates the component along the $k$ th coordinate axis, i.e., the covariance matrix is diagonal. The applications we present below in segmentation and tracking use this form which is a generalization over the original FGT algorithm. Our ongoing work (the details of which, including a proof of convergence are to be reported elsewhere) extends the variable scale FGT algorithm to sums of the form 5 .

\subsection{Speeding up computations with Gaussians}

Before describing the FGT, it is appropriate to describe some algorithmic improvements for evaluating Gaussian mixture sums proposed in the literature, which are distinct from the FGT.

The evaluation of a special function such as the exponential is usually expensive, requiring $\sim(100)$ floating point operations using the normal libraries supplied with compilers. Approximate libraries that achieve accuracy almost everywhere, and require fewer floating point operations are available, e.g., the Intel approximate math library [8] claims a speedup of a factor of 5 for evaluating exponentials over the corresponding x87 implementation. The FGT is distinct from these, and indeed the approximate math libraries can be used in conjunction with it to achieve further speed-up.

A second speed-up of gaussian sums is based on the observation that since a Gaussian typically decays relatively quickly, leading to insignificant additions when it is evaluated beyond a specified distance, heuristics can be used to avoid unnecessary evaluations when $\sigma^{-1}(|s-t|)$ is small. Some authors (e.g., Fritsch \& Rogina, 1996 [9]) have formalized this by developing a data structure that enables automating this heuristic. The FGT also employs such data structures, and automates the observation that lies behind this heuristic by providing rigorous error bounds. 


\subsection{Overview of the Algorithm}

The shifting identity that is central to the algorithm is a re-expansion of the exponential in terms of a Hermite series by using the identity

$$
e^{-\left(\frac{t-s}{\sigma}\right)^{2}}=e^{-\left(\frac{t-s_{0}-\left(s-s_{0}\right)}{\sigma}\right)^{2}}=e^{-\left(\frac{t-s_{0}}{\sigma}\right)^{2}} \sum_{n=0}^{\infty} \frac{1}{n !}\left(\frac{s-s_{0}}{\sigma}\right)^{n} H_{n}\left(\frac{t-s_{0}}{\sigma}\right),
$$

where $H_{n}$ are the Hermite polynomials. This formula tells us how to evaluate the Gaussian field $\exp \left(-\left(\frac{t-s}{\sigma}\right)^{2}\right)$ at the target $t$ due to the source at $s$, as an Hermite expansion centered at any given point $s_{0}$. Thus a Gaussian centered at $s$ can be shifted to a sum of Hermite polynomials times a Gaussian, all centered at $s_{0}$. The series converges rapidly and for a given precision only $p$ terms need to be retained. The quantities $t$ and $s$ can be interchanged to obtain a Taylor series around the target location as

$$
e^{-\left(\frac{t-s}{\sigma}\right)^{2}}=e^{-\left(\frac{t-t_{0}-\left(s-t_{0}\right)}{\sigma}\right)^{2}} \simeq \sum_{n=0}^{p} \frac{1}{n !} h_{n}\left(\frac{s-t_{0}}{\sigma}\right)\left(\frac{t-t_{0}}{\sigma}\right)^{n} .
$$

where the Hermite functions $h_{n}(t)$ are defined by

$$
h_{n}(t)=e^{-t^{2}} H_{n}(t) .
$$

The algorithm achieves its gain in complexity by avoiding evaluating every Gaussian at every evaluation point (which leads to $O(N M)$ operations). Rather, equivalent $p$ term series are constructed about a small number of source clustercenters using Equation 6 (for $O\left(N p^{d}\right)$ operations). These series are then shifted to target cluster-centers, and evaluated at the $M$ targets in $O\left(M p^{d}\right)$ operations. Here the number of terms in the series evaluations, $p$, is related to the desired level of precision $\epsilon$, and is typically small as these series converge quickly.

The process is illustrated in Figure 1. The sources and targets are divided into clusters using a simple boxing operation. This permits the division of the Gaussians according to their locations The domain is scaled to be of $O(1)$, and the box sizes are chosen to be of size $r \sqrt{2} \sigma$ where $r$ is a scale parameter.

Since Gaussians decay rapidly, sources in a given box will have no effect (in terms of the desired accuracy) to targets relatively far from there sources (in terms of distance scaled by the standard deviation $\sigma$ ). Therefore the effect of sources in a given box need to be computed only for targets in close boxes. Given the sources in one box and the targets in a neighboring box, the computation is performed using one of the following four methods depending on the number of sources and targets in these boxes: Direct evaluation is used if the number of sources and targets are small. If the sources are clustered in a box then they can be transformed into Hermite expansion about the center of the box using equation 6. This expansion is directly evaluated at each target if the number of the targets is small. If the targets are clustered then the sources or their expansion are converted to a local Taylor series (equation 7) which is then 

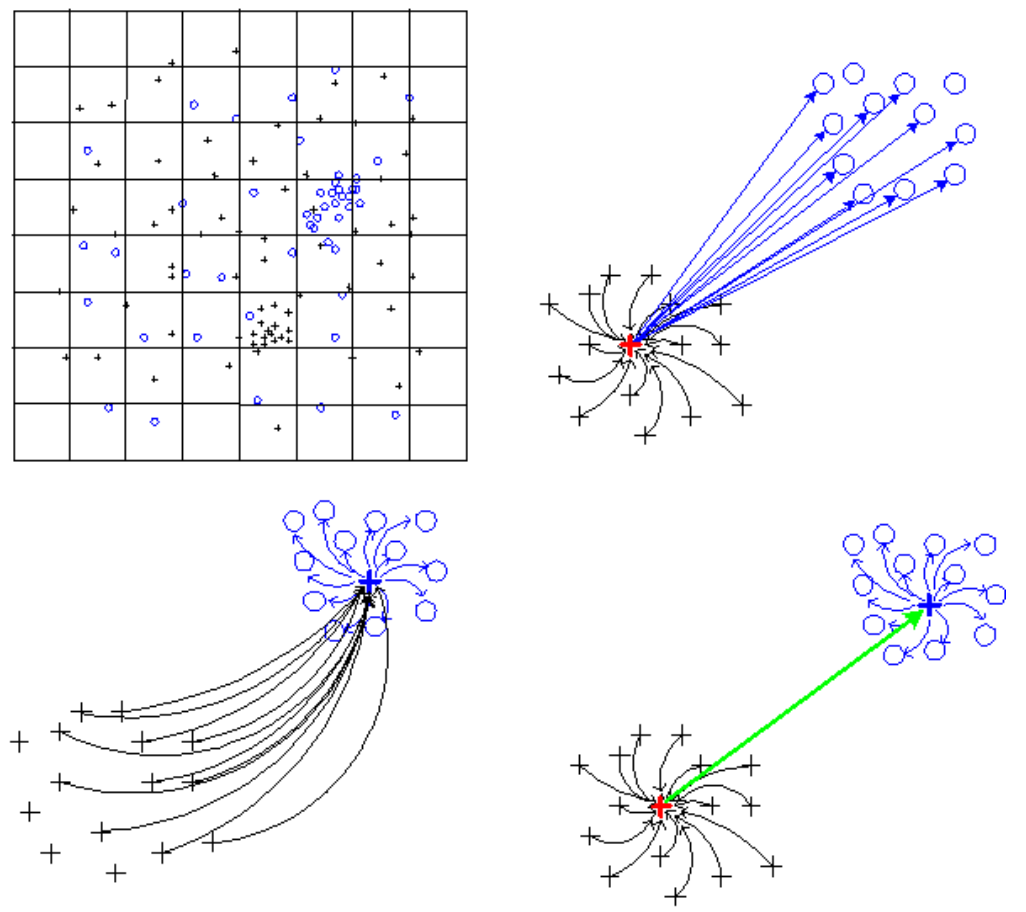

Figure 1: The fast gauss transform performs gaussian sums to a prescribed degree of accuracy by using either direct evaluations of the Gaussian for isolated sources and targets, or consolidates sources with Hermite series evaluation at isolated targets, or consolidates many sources near a clustered target location via Taylor series, or combines Hermite and Taylor series for clustered sources and targets.

evaluated at each target in the box. The number of terms to be retained in the series, $p$, depends on the required precision, the box size scale parameter $r$ and the standard deviation $\sigma$. The break-even point when using expansion is more efficient than direct evaluation is $O\left(p^{d-1}\right)$. Further details may be obtained from [4]. The clustering operation is aided by the use of appropriate data structures.

\subsection{Fast Gauss Transform Experimental Results}

Before presenting applications of the FGT to vision problems, we present some experimental results, that illustrate further how the algorithm achieves its speedup. The first experiment compares the performance of the FGT with different choices of the required precision $\epsilon$ with direct evaluation for a 2D problem. Table 1 and Figure 2 shows the CPU time using direct evaluation versus that using FGT for different precisions, $\epsilon=10^{-4}, 10^{-6}$, and $10^{-8}$ for sources with $\sigma=0.05$. 
The box size scale parameter $r$ was set to $0.5^{1}$. The sources and targets were uniformly distributed in the range $[0,1]$ and the strength of the sources were random between 0 and 1 . Table 2 shows the division of work between the different components of the FGT algorithm for the $\epsilon=10^{-6}$ case. From the division of work we notice that for relatively small number of sources and targets only direct evaluation is performed. Although, in this case, the algorithm performs only direct evaluations, it gives five to ten times speed up when compared with direct evaluation because of the way the algorithm divides the space into boxes and the locality of the direct evaluation based on the desired precision. i.e., the FGT algorithm does a smart direct evaluation. This is comparable to the speed-ups reported by Fritsch and Rogina with their bucket-box data structure. As the number of sources and targets increases and they become more clustered, other evaluation decisions are made by the algorithm, and the algorithm starts to show the linear $(O(N+M))$ performance. For very large number of sources and targets, the computation are performed through Hermite expansions of sources transformed into Taylor expansions as described above, and this yields a significant speedup. For example, for $N=M=10^{5}$, the algorithm gives more than 800 time speedup than direct evaluation for $10^{-4}$ precision.

From the figures we also note that the FGT starts to outperform direct evaluation for number of sources and targets as low as 60-80 based on the desired accuracy. This break-even point can be pushed further down by increasing the box size scale parameter, $r$. This will enhance the performance of the algorithm for small $N, M$ but will worsen the asymptotic performance.

\begin{tabular}{|l|l|l|l|l|}
\hline & Direct & \multicolumn{3}{|c|}{ Fast Gauss } \\
\cline { 3 - 5 } $\mathrm{N}=\mathrm{M}$ & Evaluation & $\epsilon=10^{-4}$ & $\epsilon=10^{-6}$ & $\epsilon=10^{-8}$ \\
\hline \hline 50 & 0.7 & 0.8 & 0.9 & 1.1 \\
100 & 2.7 & 1.3 & 1.7 & 2.0 \\
200 & 10.8 & 2.9 & 3.7 & 4.6 \\
400 & 43 & 8.2 & 10.8 & 14 \\
800 & 174 & 27 & 36 & 46 \\
1600 & 689 & 96 & 130 & 163 \\
3200 & 2754 & 319 & 493 & 640 \\
6400 & 11917 & 660 & 1281 & 1935 \\
12800 & 58500 & 942 & 2022 & 3277 \\
25600 & $234 \times 10^{3}$ & 1429 & 3210 & 5113 \\
51200 & $936 \times 10^{3}$ & 2405 & 5602 & 8781 \\
102400 & $3744 \times 10^{3}$ & 4382 & 10410 & 16181 \\
204800 & $14976 \times 10^{3}$ & 8428 & 20191 & 31106 \\
1024000 & $374 \times 10^{6}$ & 43843 & 100263 & 153791 \\
\hline
\end{tabular}

Table 1: Run time in milliseconds for direct evaluation vs. FGT with different precision

Figure 3 shows the performance of the algorithm for cases where the sources and the targets are clustered differently. The direct evaluation is compared with

\footnotetext{
${ }^{1}$ The software was written in Visual $\mathrm{C}++$ and the results were obtained on a $700 \mathrm{MHz}$ Intel Pentium III PC with 512 MB RAM.
} 


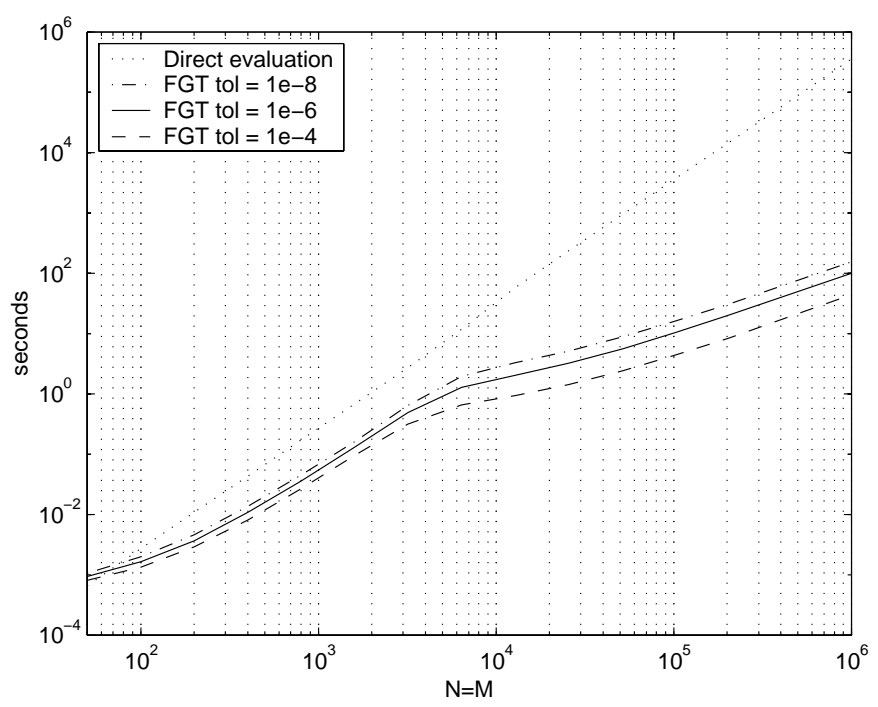

Figure 2: Run time for direct evaluation vs. FGT with different precision

\begin{tabular}{|l|l|l|l|l|}
\hline \multicolumn{5}{|c|}{ Division of work (\%) - Uniform sources and targets } \\
\hline \hline & Direct & Taylor & Hermit & Hermit + Taylor \\
$\mathrm{N}=\mathrm{M}$ & Evaluation & Expansion & Expansion & Expansion \\
\hline \hline$\leq 800$ & 100 & 0 & 0 & 0 \\
1600 & 96.6 & 1.6 & 1.8 & 0 \\
3200 & 65.7 & 15.6 & 15 & 3.7 \\
6400 & 5.3 & 18.3 & 16.9 & 59.5 \\
12800 & 0 & 0.3 & 0.3 & 99.4 \\
$\geq 25600$ & 0 & 0 & 0 & 100 \\
\hline
\end{tabular}

Table 2: Division of work between different computational methods for uniformly distributed random sources and targets

the FGT for three configurations of sources and targets: in the first case, the sources and targets are uniformly distributed between 0 and 1 . In the second case the sources are clustered inside a circle of radius 0.1 and the targets are uniformly distributed between 0 and 1 . In the third case, both the sources and the targets are clustered inside a circle of radius 0.1 . For all three cases the desired precision was set to $10^{-6}$, the sources have a scale $\sigma=0.05$ and a random strength between 0 and 1 . The division of work for the three cases are shown in Tables 2, 3, and 4. From the figures we note that for the cases where sources and/or targets are clustered the computation shows linear time behavior for number of sources and targets as low as 100, which yields a significant speedup. For a very large number of sources and targets, the computations are performed through Hermite expansions of sources transformed into Taylor expansions.

Figure 4 shows the same experiment with the three configurations of sources and targets for the $3 \mathrm{D}$ case with precision set to $10^{-6}$ and $r=0.5$. Note that the 


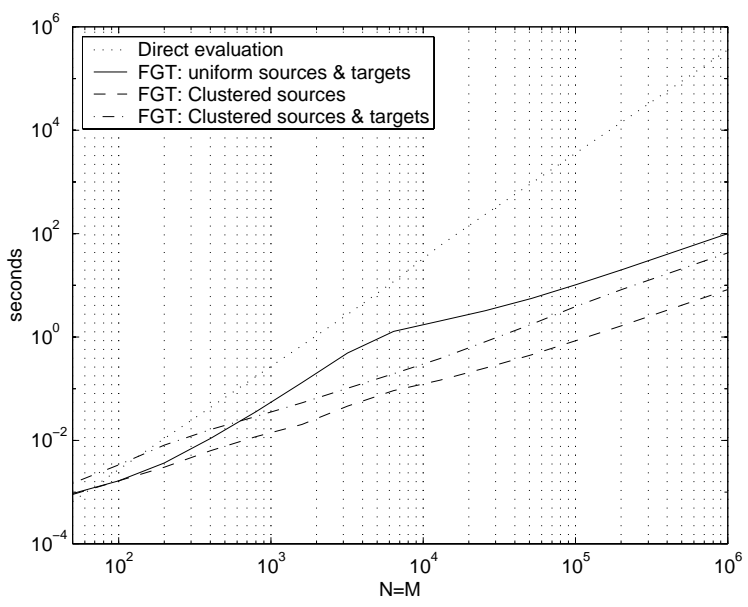

Figure 3: Run time for FGT with different configuration of sources and targets layout

\begin{tabular}{|l|l|l|l|l|}
\hline \multicolumn{4}{|c|}{ Division of work (\%) - Clustered sources, Uniform targets } \\
\hline \hline & Direct & Taylor & Hermit & Hermit + Taylor \\
$\mathrm{N}=\mathrm{M}$ & Evaluation & Expansion & Expansion & Expansion \\
\hline \hline 100 & 83.7 & 0 & 16.3 & 0 \\
200 & 65.2 & 0 & 34.8 & 0 \\
400 & 32.7 & 0 & 67.3 & 0 \\
800 & 21.3 & 0 & 78.7 & 0 \\
1600 & 17.1 & 0.3 & 81.4 & 1.2 \\
3200 & 9.2 & 2.7 & 69.2 & 18.9 \\
6400 & 1.8 & 5.6 & 22.5 & 70.1 \\
12800 & 0 & 2.6 & 0.4 & 97 \\
$\geq 25600$ & 0 & 0 & 0 & 100 \\
\hline
\end{tabular}

Table 3: Division of work between different computational methods for clustered sources and uniformly distributed targets

algorithm starts to utilize computations using Hermite and/or Taylor expansion only when the number of sources and/or targets in a box exceeds a break point of order $p^{d-1}$ which is higher in the $3 \mathrm{D}$ case. This causes the algorithm to do mostly direct evaluation for the uniform sources and targets case while Hermite and Taylor expansion computations were utilized for large number of clustered sources and/or targets.

Figure 5 shows effect of the source scale on the run time of the FGT algorithm. The figure shows the run time for three cases where sources have scale $\sigma=0.1,0.05,0.01$. For all the cases, the sources and targets were uniformly distributed between 0 and 1 . The box size scale parameter was set to $r=0.5$ for all the cases. The run time in all the cases converges asymptotically to the linear behavior. 


\begin{tabular}{|l|l|l|l|l|}
\hline \multicolumn{5}{|c|}{ Division of work (\%) - Clustered sources and targets } \\
\hline \hline & Direct & Taylor & Hermit & Hermit + Taylor \\
$\mathrm{N}=\mathrm{M}$ & Evaluation & Expansion & Expansion & Expansion \\
\hline \hline 100 & 69.4 & 13.9 & 13.9 & 2.8 \\
200 & 36.9 & 28.7 & 19.3 & 15.1 \\
400 & 12.7 & 21.6 & 24.4 & 41.3 \\
800 & 4.8 & 16.8 & 17.4 & 61.0 \\
1600 & 2.8 & 15.1 & 13.0 & 69.1 \\
3200 & 2.2 & 10.3 & 15.3 & 72.2 \\
6400 & 0.8 & 6.8 & 9.3 & 83.1 \\
12800 & 0.1 & 2.4 & 2.4 & 95.1 \\
$\geq 25600$ & 0 & 0 & 2.4 & 97.6 \\
\hline
\end{tabular}

Table 4: Division of work between different computational methods for clustered sources and targets

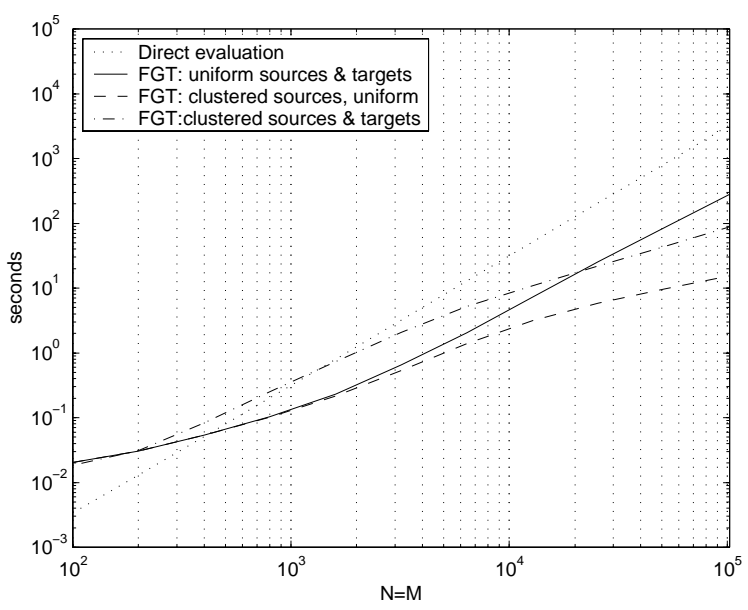

Figure 4: Run time for 3D - FGT with different configuration of sources and targets layout

\section{Region Segmentation via Color Modeling}

\subsection{Color Density Estimation}

In this application we utilize the fast Gauss algorithm for efficient density estimation of color distribution of a region. We use this approach to model the color distribution on people's clothing and to segment major body parts based on clothing. A variety of parametric and non-parametric statistical techniques have been used to model the color and the spatial properties of colored regions. In [10] the color properties of a region (blob) were modeled using a single Gaussian in the three dimensional $Y U V$ space. The use of a single Gaussian to model the color of a blob restricts the blob to be of a single color which is not a general enough assumption about the clothes people wear which usually contain 


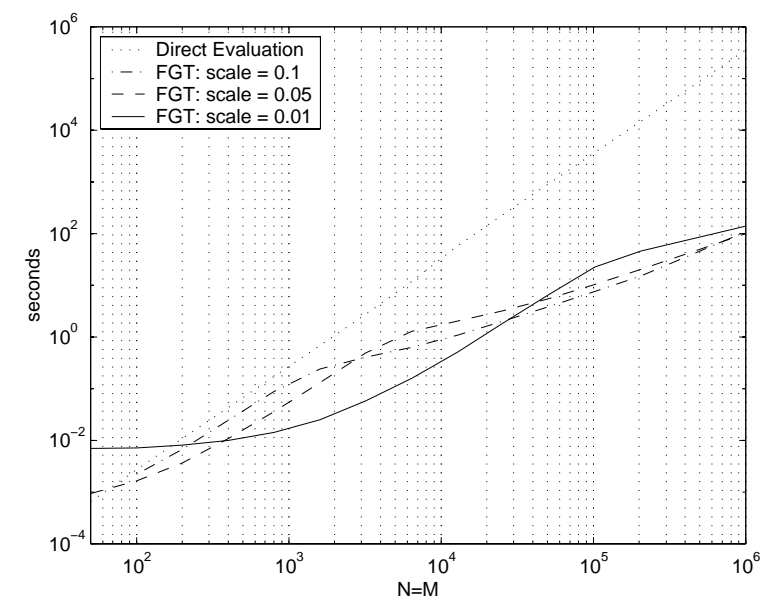

Figure 5: Run time for 2D - FGT with uniformly distributed sources and targets with different scales.

patterns and have many colors. Fitting a mixture of Gaussian using the EM algorithm provides a way to model blobs with a mixture of colors. This technique was used in $[11,12]$ for color based tracking of a single blob and was applied for tracking faces. The mixture of Gaussian technique faces the problem of choosing the right number of Gaussians for the assumed model. Non-parametric techniques using histograms have also been used for modeling color distributgions. In [13] 3-dimensional adaptive histograms in RGB space were used to model the color of the whole person. Color histograms have also been used in [14] for tracking hands. The major drawback with color histograms is the lack of convergence to the right density function if the data set is small.

We use a non-parametric approach to model the color distribution of a uniform region. Given a sample $S=\left\{x_{i}\right\}$ taken from the region where $i=1 \ldots N$ and $x_{i}$ is a $d$-dimensional vector representing the color, we can estimate the density function at any point $y$ of the color space directly from $S$ using kernel density estimation [2]

$$
\hat{P}(y)=\frac{1}{N \sigma_{1} \ldots \sigma_{d}} \sum_{i=1}^{N} \prod_{j=1}^{d} K\left(\frac{y_{j}-x_{i j}}{\sigma_{j}}\right),
$$

where the same kernel function is used in each dimension with a different bandwidth $\sigma_{j}$ for each dimension of the color space.

Theoretically, kernel density estimators can converge to any density shape with enough samples [2]. Unlike histograms, even with a small number of samples, kernel density estimation leads to a smooth continuous density estimate. If the underlying distribution is a mixture of Gaussians, kernel density estimation converges to the right density with a small number of samples. Unlike parametric fitting of a mixture of Gaussians, kernel density estimation is a more general 
approach that does not require the selection of the right number of Gaussians to be fitted. One other important advantage of using kernel density estimation is that the adaptation of the model is trivial by adding new samples.

If Gaussian kernels $K_{\sigma}(t)=(\sqrt{2 \pi} \sigma)^{-1} e^{-1 / 2(t / \sigma)^{2}}$ are used then the Fast Gauss transform computational framework gives an efficient way to compute the density estimate in equation 9 at many points, $y$, of the space in batches. In this case, each sample $x_{i}$ is considered a source point and each evaluation point, $y$, is considered as a target point. This batch evaluation is suitable in many computer vision applications since it is usually desired to evaluate the density estimate at many pixels of the image at once. Since color spaces are low in dimension, the use of fast Gauss transform will out perform direct evaluation at a low break point. Another reason that favors the use of fast gauss in color modeling applications is that the sources (the samples) are clustered in the space as well as the targets (the evaluation points).

\subsection{Color-based Body Part Segmentation}

We use the described color modeling approach to segment foreground regions corresponding to tracked people into major body parts. The foreground regions are detected using background subtraction [15]. People can be dressed in many different ways, but generally the way people are dressed leads to a set of major color regions aligned vertically (shirt, T-shirt, jacket etc., on the top and pants, shorts, skirts etc., on the bottom) for people in upright pose. We consider the case where people are dressed in a top-bottom manner which yields a segmentation of the person into a head, torso and bottom. Generally, a person in an upright pose is modeled as a set of vertically aligned blobs $M=\left\{A_{i}\right\}$ where a blob $A_{i}$ models a major color region along the vertical axis of the person representing a major part of the body as the torso, bottom or head. Each blob is represented by its color distribution as well as its spatial location with respect to the whole body. Since each blob has the same color distribution everywhere inside the blob and since the vertical location of the blob is independent of the horizontal axis, the joint distribution of pixel $(x, y, c)$ (the probability of observing color $c$ at location $(x, y)$ given blob $A$ ) is a multiplication of three density functions [16].

$$
P_{A}(x, y, c)=f_{A}(x) g_{A}(y) h_{A}(c),
$$

where $h_{A}(c)$ is the color density of blob $A$ and the densities $g_{A}(y), f_{A}(x)$ represents the vertical and horizontal location of the blob respectively.

Estimates for the color density $h_{A}(c)$ can be calculated using the kernel density estimation as was shown in 9 . We represent the color of each pixel as a 3-dimensional vector $X=(r, g, s)$ where $r=\frac{R}{R+G+B}, g=\frac{G}{R+G+B}$ are two chromaticity variables and $s=(R+G+B) / 3$ is a lightness variable. Given a sample of pixels $S_{A}=\left\{X_{i}=\left(r_{i}, g_{i}, s_{i}\right)\right\}$ from blob $A$, an estimate $\hat{h}_{A}(\cdot)$ for the 
color density $h_{A}(\cdot)$ can be calculated as

$$
\hat{h}_{A}(r, g, s)=\frac{1}{N} \sum_{i=1}^{N} K_{\sigma_{r}}\left(r-r_{i}\right) K_{\sigma_{g}}\left(g-g_{i}\right) K_{\sigma_{s}}\left(s-s_{i}\right),
$$

where $K_{\sigma}(t)=1 / \sigma K(t / \sigma)$. Using Gaussian kernels, i.e., $K_{\sigma}(t)=(\sqrt{2 \pi} \sigma)^{-1} e^{-1 / 2(t / \sigma)^{2}}$ with different bandwidths in each dimension, the density estimation can be evaluated as a sum of Gaussians as

$$
\hat{h}_{A}(r, g, s)=\frac{1}{N} \cdot C \sum_{i=1}^{N} e^{-1 / 2\left(\frac{r-r_{i}}{\sigma_{r}}\right)^{2}} e^{-1 / 2\left(\frac{g-g_{i}}{\sigma_{g}}\right)^{2}} e^{-1 / 2\left(\frac{s-s_{i}}{\sigma_{s}}\right)^{2}}
$$

where $C$ is a constant. At each new frame, it is desired to evaluate this probability at different locations. The FGT algorithm is used to efficiently compute these probabilities. Here the sources are the sample locations $S_{A}$, while the targets are the vectors $(r, g, s)$ at each evaluation location. The estimation of the bandwidths for each dimension is done offline by considering batches of regions with a single color distribution taken from images of people's clothing and estimating the variance in each color dimension. This model is not restricted to a particular color space, and can be extended to any three dimensional color space.

Given a set of samples $S=\left\{S_{A_{i}}\right\}$ corresponding to each blob and initial estimates for the position of each blob $y_{A_{i}}$, each pixel is classified into one of the three blobs based on maximum likelihood classification assuming that all blobs have the same prior probabilities

$$
\begin{aligned}
X \in A_{k} \text { s.t. } k & =\arg _{k} \max P\left(X \mid A_{k}\right) \\
& =\arg _{k} \max g_{A_{k}}(y) h_{A_{k}}(c)
\end{aligned}
$$

where the vertical density $g_{A_{k}}(y)$ is assumed to have a Gaussian distribution $g_{A_{k}}(y)=N\left(y_{A_{k}}, \sigma_{A_{k}}\right)$. Since the blobs are assumed to be vertically above each other, the horizontal density $f_{A}(x)$ is irrelevant to the classification.

A horizontal blob separator is detected between each two consecutive blobs by finding the horizontal line that minimizes the classification error. Given the detected blob separators, the color model is recaptured be sampling pixels from each blob. Blob segmentation is performed and blob separators are detected at each new frame as long as the target is isolated and tracked.

Model initialization is done automatically by taking three samples $S=$ $\left\{S_{H}, S_{T}, S_{B}\right\}$ of pixels from three confidence bands corresponding to the head, torso and bottom. The locations of these confidence bands are learned offline as follows: A set of training data ${ }^{2}$ is used to learn the location of blob separators (head-torso, torso-bottom) with respect to the body for a set of people in upright pose where these separators are manually marked. Figure 6-a shows

\footnotetext{
${ }^{2}$ The training data consists of 90 samples of different people from both genders in different orientations in upright pose.
} 

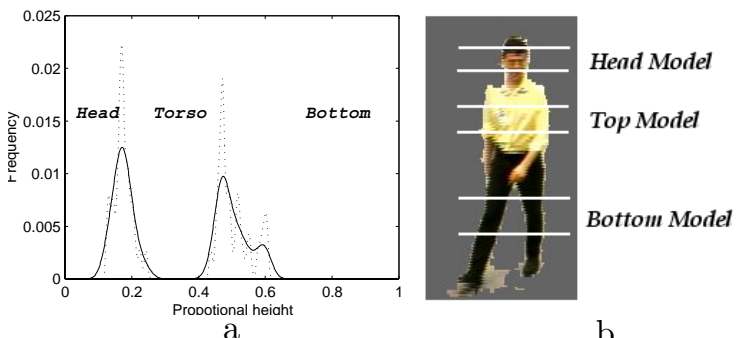

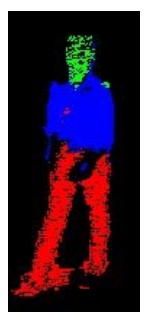

$\mathrm{C}$

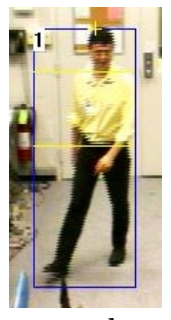

d

Figure 6: a- Blob separator histogram from training data. b- Confidence bands. c - Blob segmentation. d- Detected blob separators

a histogram of the locations of head-torso (left peak) and torso-bottom (right peak) in the training data. Based on these separator location estimates, we can determine the confidence bands proportional to the height where we are confident that they belong to head, torso and bottom and use them to capture initial samples $S=\left\{S_{H}, S_{T}, S_{B}\right\}$. Figure 6 -b shows initial bands used for initialization where the segmentation result is shown in 6-c and the detected separators are shown in 6-d.
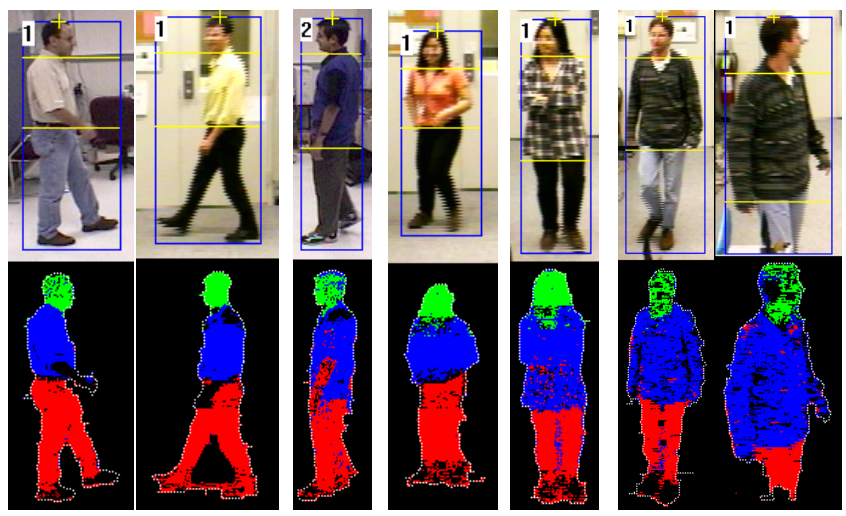

Figure 7: Example results for blob segmentation

Figure 7 illustrates some blob segmentation examples for various people. Notice that the segmentation and separator detection is robust even under partial occlusion of the target as in the rightmost result. Also in some of these examples the clothes are not of a uniform color. In [16] we showed how this representation can be used to segment foreground regions corresponding to multiple people in occlusion. 


\section{People Tracking Application}

In this application we show how the fast Gauss transform algorithm can be used to efficiently compute estimate for the gradient of the density as well as estimate for the density itself. Estimation of density gradient is useful in applications that use the mean shift algorithm [17, 18, 19, 20]. We utilize the color-spatial joint distribution of a region as a constraint that is used to locate that region in subsequent frames and therefore to track this region. The color-spatial joint density is estimated using kernel density estimation. An estimate of the gradient of the joint density is used to drive a gradient based search that is used to track that region. In order to reduce the dimensionality of the problem, we use one spatial dimension and two color dimensions to represent the joint color-spatial density. This is justified, since for the majority of clothing for people in upright pose, the color distribution is independent of the horizontal axis and depends only on the vertical axis. That is, we are most likely to observe the same color distribution orthogonal to the vertical axis. (Note that this does not assume constant horizontal color). This allows us to use only the vertical dimension in our representation of the joint color-spatial density. The independence of the color distribution from the abscissa makes the representation robust to rotation in depth.

Let $S=\left\{X_{i}\right\}_{i=1 . . n}$ be a set of sample pixels from the target region so that $X_{i}=\left(x_{i}, y_{i}, u_{i}\right)$ where $x_{i}, y_{i}$ is the sample pixel location around an origin $\mathbf{o}$, and $u_{i} \in R^{d}$ is a $d$-dimensional color vector. Given the sample $S$ as a model for the target region, the joint spatial-color probability of observing a pixel $X$ at location $(x, y)$ with color $u$ can be expressed as two independent density functions (since the color distribution is assumed to be independent of the horizontal axis and depends only on the vertical axis)

$$
P(x, y, u)=F(x) H(y, u),
$$

where $H(y, u)$ represents the joint density between the vertical location and the color features and $F(x)$ represents the distribution along the horizontal axis. If we use two chromaticity variables $u=\left(u_{1}, u_{2}\right)$ with diagonal covariance matrix to represent the color for each pixel, then we can estimate joint density $\hat{H}(y, u \mid S)$ as:

$$
\hat{H}(y, u)=\frac{1}{N} \sum_{i=1}^{N} K_{\sigma_{y}}\left(y-y_{i}\right) K_{\sigma_{u_{1}}}\left(u_{1}-u_{i 1}\right) K_{\sigma_{u_{2}}}\left(u_{2}-u_{i 2}\right),
$$

where $\sigma_{y}, \sigma_{u_{1}}, \sigma_{u_{2}}$ are the kernel bandwidths in the three dimensions. Given a translation of the region origin to a location $\left(x_{c}, y_{c}\right)$ and a scaling $h_{c}$, we can estimate the probability of a pixel $X=(x, y, u)$ coming from $S$ by shifting and scaling as

$$
\hat{H}_{\left(y_{c}, h_{c}\right)}(y, u)=\frac{1}{N} \sum_{i=1}^{N} K_{\sigma_{y}}\left(\frac{y-y_{c}}{h_{c}}-y_{i}\right) K_{\sigma_{u_{1}}}\left(u_{1}-u_{i 1}\right) K_{\sigma_{u_{2}}}\left(u_{2}-u_{i 2}\right) .
$$


Let $R(c)$ be a region that represents the target with respect to a location and a scaling hypothesis $c=\left(x_{c}, y_{c}, h_{c}\right)$, we define the likelihood function $\psi(c)$ by integrating $\log$ probabilities over $R$ as:

$$
\psi(c)=\int_{R} w_{c}(x, y) L\left(\hat{P}_{c}(x, y, u)\right) d x
$$

where $\hat{P}_{c}(x, y, u)$ is an estimate of the joint density in $11, L(\cdot)$ is a log function and $w_{c}(x, y)$ is a weight kernel. Practically, we can assign more weights to pixel inside the region and less weights for pixels on the region boundary since their inclusion in $R$ is expected to be noisy. The target localization at frame $t$ can be formalized as a search for a 2-D translation and a scaling that maximizes $\psi(c)$. i.e., the location and scale hypothesis $c=\left(x_{c}, y_{c}, h_{c}\right)$ that maximizes $\psi(c)$ :

$$
c=\arg _{c} \max \psi(c)
$$

Given the joint density in equation 11 the likelihood function can be written as

$$
\psi(c)=\sum_{(x, y, u) \in R} w_{c}(x, y) L\left(F_{c}(x)\right)+w_{c}(x, y) L\left(\hat{H}_{c}(y, u)\right)
$$

We can drop the first term since this will be the same for all hypotheses, and so we can redefine $\psi($.$) in a simpler way as$

$$
\psi(c)=\sum_{(x, y, u) \in R} w_{c}(x, y) L\left(\hat{H}_{c}(y, u)\right)
$$

For the weights $w_{c}(x, y)$, we use Gaussian kernels $N\left(x_{c}, \alpha_{x} h_{c}\right), N\left(y_{c}, \alpha_{y} h_{c}\right)$ where $\alpha_{x}, \alpha_{y}$ are scaling constants in the horizontal and vertical direction.

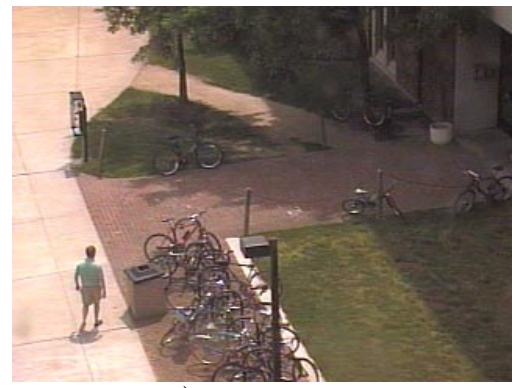

a) original image

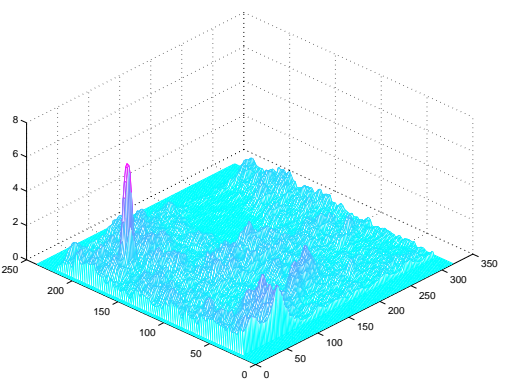

b) plot of surface $\psi(c)$

Figure 8: Likelihood function surface

The optimal target hypothesis represents a local maxima in the discrete three dimension search space. The log likelihood function is continuous and differentiable and, therefore, a gradient based optimization technique would converge to that solution as long as the initial guess is within a small neighborhood of that maxima. Figure 8 shows a plot of the function $\psi(c)$ for each location in 
the image as a hypothesis for the target origin. For this plot, $R$ was defined as a vertical ellipse of the same size as the target.

The derivation of the gradient of the objective function yields (after some manipulation):

$$
\begin{aligned}
\left(\alpha_{x} h\right)^{2} \cdot \frac{\nabla_{x} \psi(c)}{\psi(c)} & =\frac{\sum_{(x, y)} x w_{c}(x, y) L\left(\hat{H}_{c}\right)}{\sum_{(x, y)} w_{c}(x, y) L\left(\hat{H}_{c}\right)}-x_{c} \\
\left(\alpha_{y} h\right)^{2} \cdot \frac{\nabla_{y} \psi(c)}{\psi(c)} & =\left(\left(\alpha_{y} h\right)^{2} \frac{\sum_{(x, y)} w_{c}(x, y) \frac{\nabla_{y} \hat{H}_{c}}{\hat{H}_{c}}}{\sum_{(x, y)} w_{c}(x, y) L\left(\hat{H}_{c}\right)}\right. \\
& \left.+\frac{\sum_{(x, y)} y w_{c}(x, y) L\left(\hat{H}_{c}\right)}{\sum_{(x, y)} w_{c}(x, y) L\left(\hat{H}_{c}\right)}\right)-y_{c}
\end{aligned}
$$

where the term $\frac{\nabla_{y} \hat{H}_{c}}{\hat{H}_{c}}$ is the normalized gradient of the joint density $H_{c}$ which can be derived from equation 12 and can be expressed as

$$
\begin{aligned}
& \frac{\nabla_{y} \hat{H}_{c}(x, y, u)}{\hat{H}_{c}(x, y, u)}=\frac{-1}{\sigma^{2} h} . \\
& \left(\frac{\sum_{i=1}^{N} x_{i} K_{\sigma_{y}}\left(\frac{y-y_{c}}{h_{c}}-y_{i}\right) K_{\sigma_{u_{1}}}\left(u_{1}-u_{i 1}\right) K_{\sigma_{u_{2}}}\left(u_{2}-u_{i 2}\right)}{\sum_{i=1}^{N} K_{\sigma_{y}}\left(\frac{y-y_{c}}{h_{c}}-y_{i}\right) K_{\sigma_{u_{1}}}\left(u_{1}-u_{i 1}\right) K_{\sigma_{u_{2}}}\left(u_{2}-u_{i 2}\right)}-\frac{y-y_{c}}{h_{c}}\right)
\end{aligned}
$$

The main computation overhead is the calculation of the joint density estimate $\hat{H}_{c}\left(y, u_{1}, u_{2}\right)$ and its gradient, $\nabla_{y} \hat{H}_{c}(x, y, u)$. That is, the evaluation of Gaussian summation

$$
S_{1}\left(\dot{y}, u_{1}, u_{2}\right)=C_{1} \sum_{i=1}^{N} e^{-\frac{1}{2}\left(\frac{\dot{y}-y_{i}}{\sigma y}\right)^{2}} e^{-\frac{1}{2}\left(\frac{u_{1}-u_{i 1}}{\sigma u_{1}}\right)^{2}} e^{-\frac{1}{2}\left(\frac{u_{2}-u_{i 2}}{\sigma u_{2}}\right)^{2}}
$$

and a weighted version of it

$$
S_{2}\left(\dot{y}, u_{1}, u_{2}\right)=C_{2} \sum_{i=1}^{N} y_{i} e^{-\frac{1}{2}\left(\frac{\dot{y}-y_{i}}{\sigma y}\right)^{2}} e^{-\frac{1}{2}\left(\frac{u_{1}-u_{i 1}}{\sigma u_{1}}\right)^{2}} e^{-\frac{1}{2}\left(\frac{u_{2}-u_{i 2}}{\sigma_{u_{2}}}\right)^{2}}
$$

Where $C_{1}, C_{2}$ are normalization constants and $N$ is the number of samples in the target model. At each iteration We need to evaluate these sums at each pixel $\left(x, y, u_{1}, u_{2}\right)$ in the region defined by each new region origin hypothesis $c=\left(x_{c}, y_{c}, h_{c}\right)$ where $\dot{y}=\frac{y-y_{c}}{h_{c}}$. These summation is evaluated using Fast Gauss algorithm where the sources are the sample points $\left(y_{i}, u_{i 1}, u_{i 2}\right)_{i=1 . . n}$ and the targets are the evaluation pixels $\left(y, u_{1}, u_{2}\right)$ in candidate region $R(c)$. Notice that in the second summation each source is assigned a strength $y_{i} / \sum_{i=1}^{N} y_{i}$

To achieve efficient implementation, we developed a two phase version of the fast Gauss algorithm where in the first phase all the source data structures and expansions are calculated from the samples. Then, at each new frame, these 


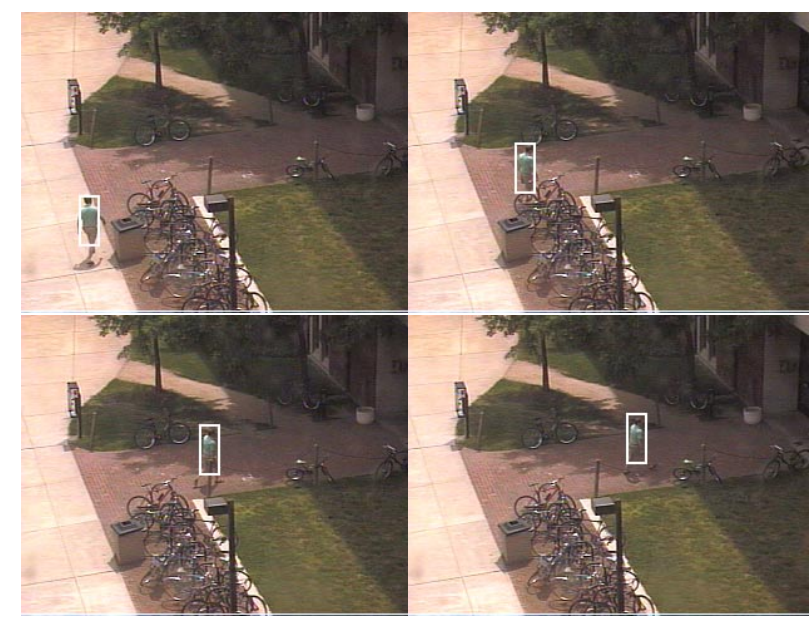

Figure 9: Tracking result

structures and expansions are reused to evaluate the new targets. Since targets (evaluation points) are expected to be similar from frame to frame, the results of each evaluation are kept in a look up table to avoid doing repeated computation.

Figure 9 shows four frames from the tracking result for a sequence. The sequence contains 40 frames taken at about 5 frames per second rate. The tracker successfully locate the target at this low frame rate. Notice that the target changes his orientation during the tracking. Figure 10-left shows the performance of the tracker on this sequence using Fast Gauss only (no look up tables). The average run-time per frame is about 200-300 msec. Figure 10-right shows the performance of the tracker with look up tables used in conjunction with fast Gauss transform to save repeated computation. In this case, the run time decreases with time and the average run time per frame is less than 20 msec. This is more than 10 times speed up per iteration.

Figure 11 shows examples of tracking under partial occlusion. The target in this case is waiting for the elevator and is not moving significantly. The results shows that the tracker continued to locate the target successfully throughout several significant occlusion situation. Notice also that the horizontal location of the located region is not affected if some parts of the body are occluded. Since there is no significant motion in most of these sequence, motion based tracking would fail in such a sequence. Also algorithms based on adaptive background subtraction might adapt to the target. Figure 12 shows results of tracking a person in a crowd. These results are for videos captured at 10-12 frame per second. The tracker successfully locates the targets at this low frame rate. The bandwidths for the joint distribution kernels were set to $5 \%, 1 \%, 1 \%$ of each dimension space size for $\sigma_{y}, \sigma_{u 1}$ and $\sigma_{u 1}$ respectively. The weight kernel $w$ is narrow in the horizontal direction $\left(\alpha_{x}=0.5 \cdot \frac{\text { width }}{2}\right)$ and wide in the vertical direction $\left(\alpha_{y}=1.8 \cdot \frac{\text { height }}{2}\right)$. Figure 13 shows a plot of the number of iterations 

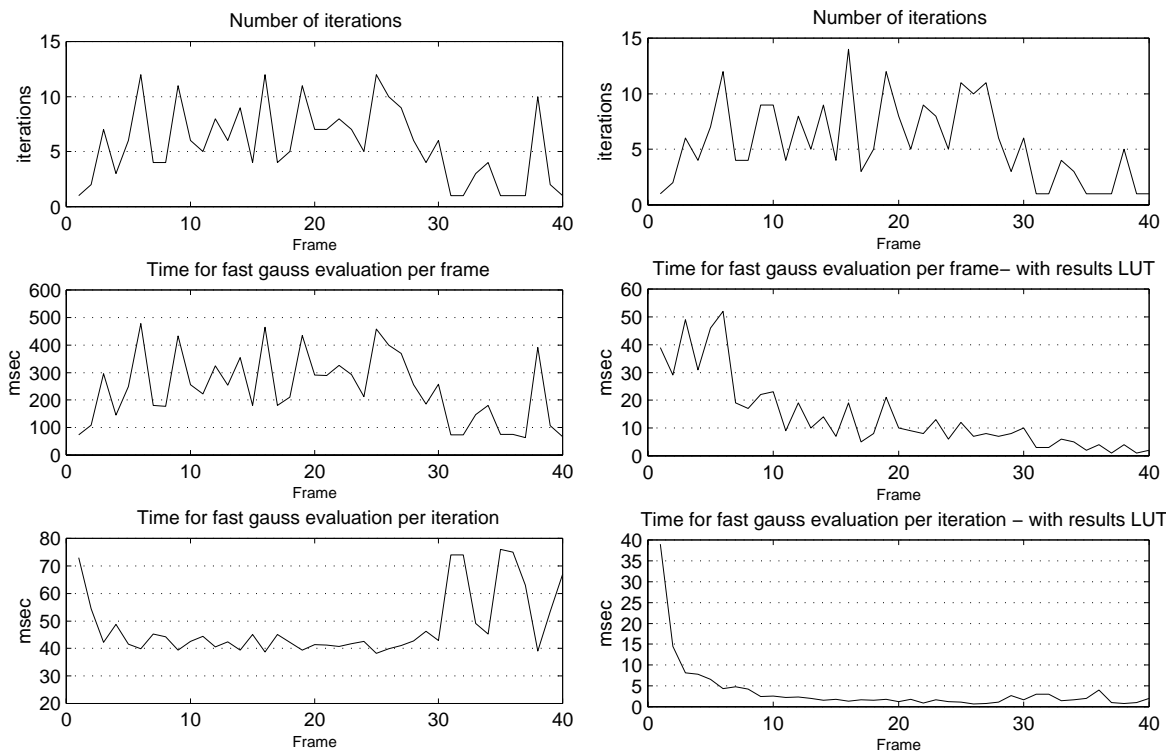

Figure 10: Performance of the tracker using Fast Gauss. Top: number of iterations per frame. Middle:run time per frame. Bottom: run time per iteration. Right: Performance using FGT with results look up tables.

needed for convergence for the sequence shown in figure 12 .

\section{Conclusion}

In this paper we investigate the use of Fast Gauss Transform to speedup kernel density estimation techniques. We presented the use of kernel density estimation in modeling the color of homogeneous regions and used this modeling approach to segment foreground region corresponding to people into major body parts based on color. We also presented the use of kernel estimation of the joint colorspatial density in tracking people. The tracking algorithm is robust to partial occlusion and rotation in depth. It can be used from moving or stationary platforms. In both applications, the FGT was used to efficiently compute the kernel density estimation and the gradient of the density, resulting in real-time performance. The Fast Gauss transform can be useful to many vision problems that use kernel density estimation, and as such our paper serves to introduce the algorithm to this community. Kernel density estimation is a better way to estimate the densities in comparison with classical parametric methods in cases where the underlying distribution are not known or when it is hard to specify the right model. Also kernel density estimation is better than use of histogram techniques, since a smooth and continuous estimate of the density is obtained, and the method has significantly improved memory complexity. The version of 


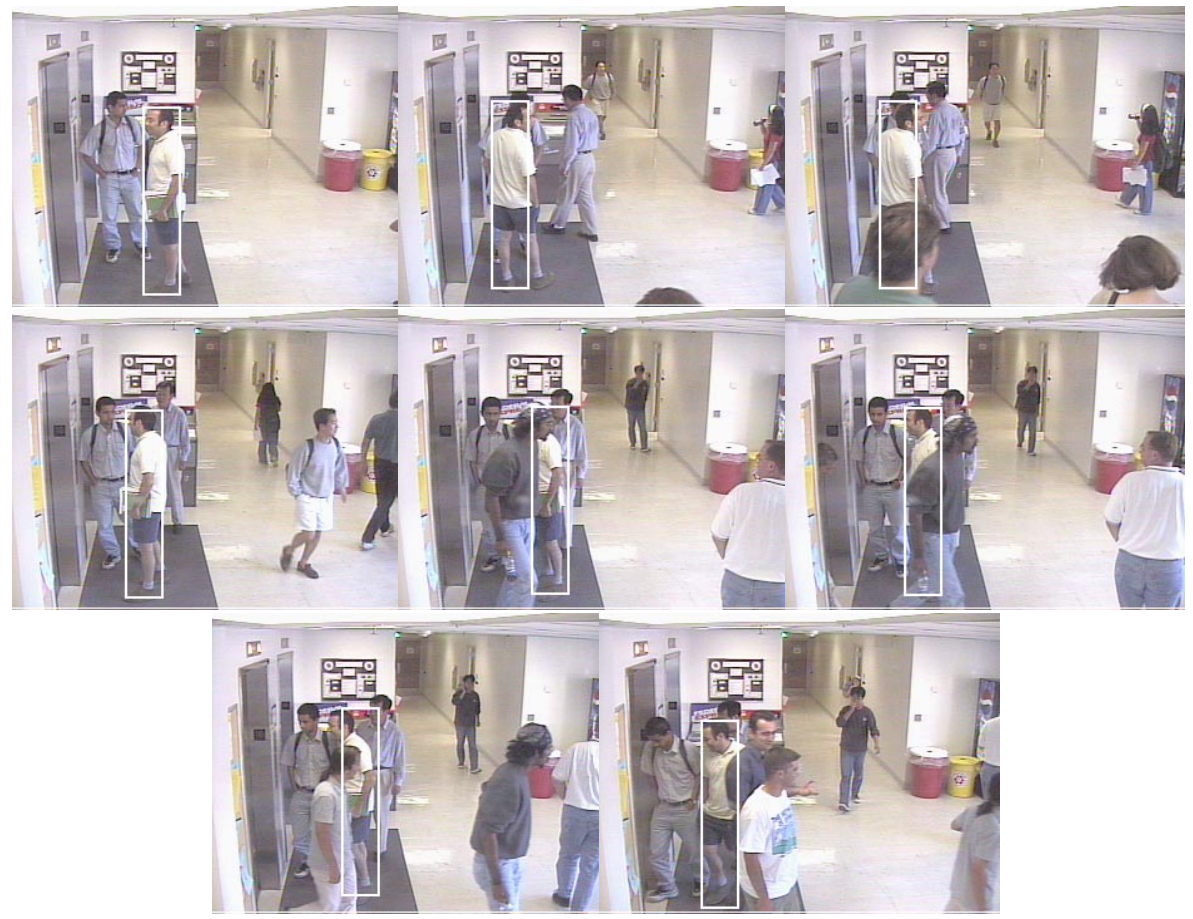

Figure 11: Tracking under partial occlusion

the FGT algorithm that we presented here is a generalization of the original algorithm as it uses a diagonal covariance matrix instead of a scalar variance. Similar generalizations to the variable scale case will be reported elsewhere. 


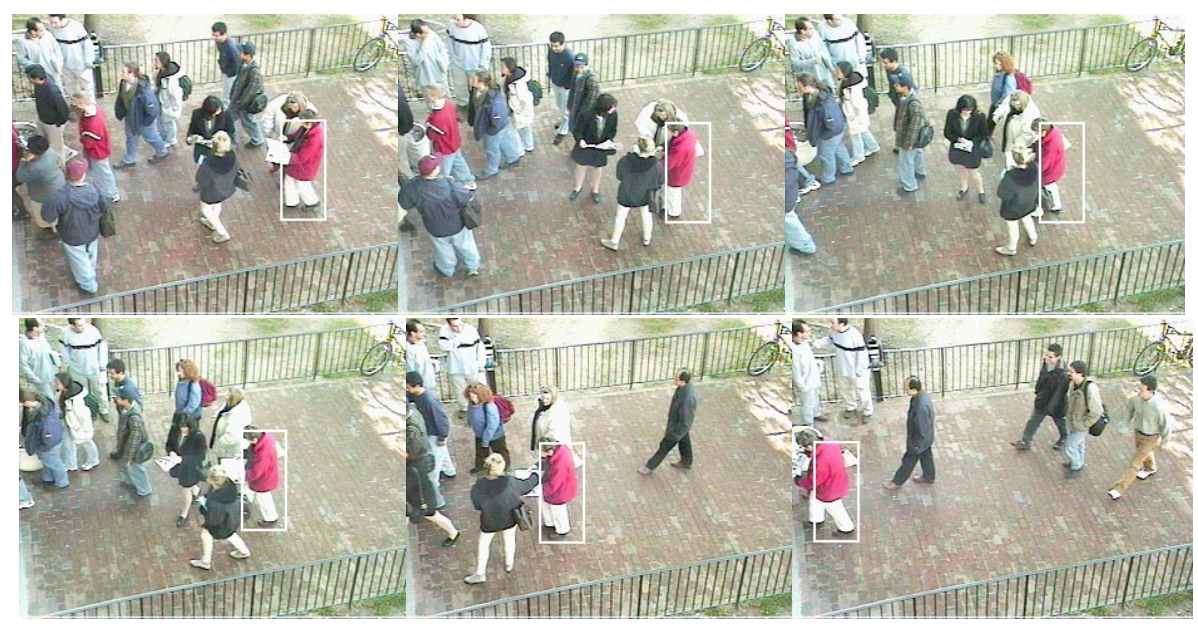

Figure 12: Tracking person in a crowd

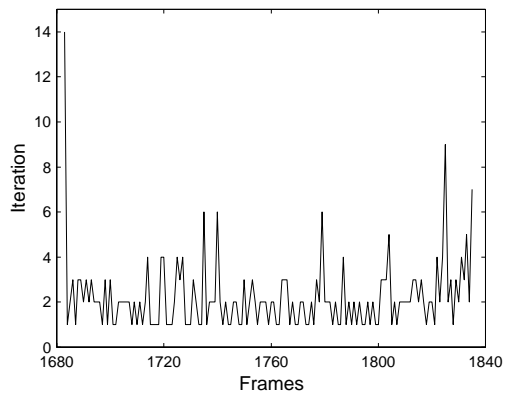

Figure 13: Number of iterations for convergence 


\section{References}

[1] R. O. Duda, D. G. Stork, and P. E. Hart, Pattern Classification. Wiley, John \& Sons, 2000.

[2] D. W. Scott, Mulivariate Density Estimation. Wiley-Interscience, 1992.

[3] L. Greengard, The Rapid Evaluation of Potential Fields in Particle Systems. Cambridge, MA: MIT Press, 1988.

[4] L. Greengard and J. Strain, "The fast gauss transform," SIAM J. Sci. Comput., 2, pp. 79-94, 1991.

[5] A. Ware, "Fast approximate fourier transforms for irregularly spaced data," SIAM Review, vol. 40, pp. 838-856, December 1998.

[6] C. Lambert, S. Harrington, C. Harvey, and A. Glodjo, "Efficient on-line nonparametric kernel density estimation," Algorithmica, no. 25, pp. 37-57, 1999.

[7] J. Strain, "The fast gauss transform with variable scales," SIAM J. Sci. Comput., vol. 12, pp. 1131-1139, 1991.

[8] "Approximate math library for intel streaming simd extensions, release 2.0." October 2000 Documentation File, Intel Corporation.

[9] J. Fritsch and I. Rogina, "The bucket box intersection (bbi) algorithm for fast approximative evaluation of diagonal mixture gaussians," in Procedings of the ICASSP 96, May 2-5, Atlanta, Georgia USA, 1996.

[10] C. R. Wern, A. Azarbayejani, T. Darrell, and A. P. Pentland, "Pfinder: Real-time tracking of human body," IEEE Transaction on Pattern Analysis and Machine Intelligence, 1997.

[11] Y. Raja, S. J. Mckenna, and S. Gong, "Colour model selection and adaptation in dynamic scenes," in 5th European Conference of Computer Vision, 1998.

[12] Y. Raja, S. J. Mckenna, and S. Gong, "Tracking colour objects using adaptive mixture models," Image Vision Computing, no. 17, pp. 225-231, 1999.

[13] S. J. McKenna, S. Jabri, Z. Duric, and A. Rosenfeld, "Tracking groups of people," Computer Vision and Image Understanding, no. 80, pp. 42-56, 2000 .

[14] J. Martin, V. Devin, and J. Crowley, "Active hand tracking," in IEEE International Conference on Automatic Face and Gesture Recognition, 1998.

[15] A. Elgammal, D. Harwood, and L. S. Davis, "Nonparametric background model for background subtraction," in 6th European Conference of Computer Vision, 2000. 
[16] A. Elgammal and L. S. Davis, "Probabilistic framework for segmenting people under occlusion," in 8th IEEE International Conference on Computer Vision, 2001.

[17] Y. Cheng, "Mean shift, mode seeking, and clustering," IEEE Transaction on Pattern Analysis and Machine Intelligence, vol. 17, pp. 790-799, Aug 1995.

[18] Y. Cheng and K. Fu, "Conceptual clustering in knowledge organization," IEEE Transaction on Pattern Analysis and Machine Intelligence, vol. 7, pp. 592-598, 1985.

[19] D. Comaniciu, V. Ramesh, and P. Meer, "Real-time tracking of non-rigid objects using mean shift," in IEEE Conference on Computer Vision and Pattern Recognition, vol. 2, pp. 142-149, Jun 2000.

[20] D. Comaniciu and P. Meer, "Mean shift analysis and applications," in IEEE 7th International Conference on Computer Vision, vol. 2, pp. 1197-1203, Sep 1999. 